\title{
Knockdown of SNW1 ameliorates brain microvascular endothelial cells injury by inhibiting NLRP3 inflammasome activation
}

\section{Type}

Research paper

\section{Keywords}

NLRP3 inflammasome, SNW1, brain microvascular endothelial cells

\begin{abstract}
Introduction

SNW domain containing 1 (SNW1), as a splicing factor to regulate the activity of transcription factors, has been reported to be involved in multiple disease processes, including neuroblastoma. Whereas, the latent function and concrete mechanism of SNW1 in brain microvascular endothelial cells (BMECs) have not been clarified.

\section{Material and methods}

BMECs were induced by oxidized low-density lipoprotein (ox-LDL), and high fat (HF)-fed rats were established. After SNW1 knockdown or NLR family pyrin domain containing 3 (NLRP3) overexpression, SNW1 and NLRP3 expressions were monitored via RT-qPCR, Western blot, or immunohistochemistry assays. Also, cell viability, apoptosis, and cholesterol efflux were determined via CCK-8, flow cytometry, and related kits; IL-18 and IL-3 levels were also certified by ELISA kits; and NLRP3 inflammasomes and cholesterol efflux-related proteins were identified by Western blot in vitro and in vivo.
\end{abstract}

\section{Results}

We discovered that ox-LDL or HF-feeding significantly elevated SNW1 and NLRP3 expressions, and prominently induced BMECs injury in BMECs or rat brain tissues. Subsequently, our data confirmed that SNW1 knockdown markedly accelerated cholesterol efflux and viability, and prevented apoptosis and NLRP3 inflammasomes, which also could be reversed by NLRP3 overexpression in ox-LDLinduced BMECs. In addition, we showed that SNW1 knockdown could signally induce cholesterol efflux and repress NLRP3 inflammasome activation in HF-fed rats.

\section{Conclusions}

We demonstrated that SNW1 knockdown has a great protection effect on the dysfunction of BMECs by inhibiting NLRP3. So, SNW1 might be a therapeutic target for BMECs injury. 
Knockdown of SNW1 ameliorates brain microvascular endothelial cell injury by inhibiting NLRP3 inflammasome activation

Running title: SNW1 in brain microvascular endothelial cells

\begin{abstract}
:
Introduction: SNW domain containing 1 (SNW1), as a splicing factor to regulate the activity of transcription factors, has been reported to be involved in multiple disease processes, including neuroblastoma. Whereas, the latent function and concrete mechanism of SNW1 in brain microvascular endothelial cells (BMECs) have not been clarified.
\end{abstract}

Material and methods: BMECs were induced by oxidized low-density lipoprotein (ox-LDL), and high fat (HF)-fed rats were established. After SNW1 knockdown or NLR family pyrin domain containing 3 (NLRP3) overexpression, SNW1 and NLRP3 expressions were monitored via RT-qPCR, Western blot, or immunohistochemistry assays. Also , cell viability, apoptosis, and cholesterol efflux were determined via CCK-8, flow cytometry, and related kits; IL-18 and IL-3 levels were also certified by ELISA kits; and NLRP3 inflammasomes and cholesterol efflux-related proteins were identified by Western blot in vitro and in vivo. 
Results: We discovered that ox-LDL or HF-feeding significantly elevated SNW1 and NLRP3 expressions, and prominently induced BMECs injury in BMECs or rat brain tissues. Subsequently, our data confirmed that SNW1 knockdown markedly accelerated cholesterol efflux and viability, and prevented apoptosis and NLRP3 inflammasomes, which also could be reversed by NLRP3 overexpression in ox-LDL-induced BMECs. In addition, , we showed that SNW1 knockdown could signally induce cholesterol efflux and repress NLRP3 inflammasome activation in HF-fed rats.

Conclusions: We demonstrated that SNW1 knockdown has a great protection effect on the dysfunction of BMECs by inhibiting NLRP3. So, SNW1 might be a therapeutic target for BMECs injury.

Key words: SNW1, brain microvascular endothelial cells, NLRP3 inflammasome

\section{Introduction}

Cerebrovascular disease (CVD) is a localized or diffuse neurological dysfunction caused by cerebral vascular wall lesions, and changes in blood composition or hemodynamics[1]. It was reported that major risk factors for CVD include hypertension, smoking, hypercholesterolemia, inflammation, diabetes, overweight/obesity, carotid arteriostenosis, metabolic syndrome, and hyperhomocysteinemia [2, 3]. With the development of neuroimaging, neurointervention, and microneurosurgery, although the diagnosis and treatment of CVD have greatly improved, it remains one of the leading causes of death and disability in humans[4]. According to statistics, there are about 2.7 million new cases of CVD and 1.3 million deaths in China every year[5]. Currently, the 
therapeutic drugs for CVD mainly include anti-platelet aggregation drugs, statins, blood-activating and blood-stasis removing drugs, free radical scavenging agents, and other drugs[6]. Moreover, some innovative therapies such as liraglutide can reduce and potentially prevent cardiovascular events[7-9]. Although these drugs have some effects, their side effects should not be ignored. Therefore, how to effectively prevent and treat CVD remains a major public health problem.

The blood-brain barrier (BBB) is a unique structure of the central nervous system (CNS) and a necessary structure to maintain the stability of the brain environment and to avoid the invasion of harmful substances into brain tissue[10, 11]. The BBB mainly consists of brain microvascular endothelial cells (BMECs) tight junction, vascular endothelial cells in CNS, and processes and basal membranes of the perivascular glial cells $[11,12]$. BMECs, as the main structure of the BBB, contribute to the exchange of substances inside and outside heart tissue in the central nervous system[13]. Researches proved that BMECs injury was closely associated with multiple diseases, such as ischemic stroke, neurodegenerative disease, and atherosclerosis [14-17]. Alao, changes in brain microvascular structure and function have been demonstrated to trigger and aggravate related neuronal injury[17, 18]. Therefore, further exploration of the molecular mechanism of functional changes of BMECs can provide a target for the therapy of cerebrovascular diseases.

Oxidized low-density lipoprotein (ox-LDL), as a predominant risk factor of atherosclerosis, has been reported to participate in the progression of cerebrovascular 
disorders[19-21]. Ox-LDL participates in the process of cerebrovascular diseases through various mechanisms, for example, Ox-LDL causes vascular endothelial injury, increases the permeability of the BBB [22], and

may serve as an indicator to reflect the level of oxidative stress[21]. Therefore, ox-LDL has been widely used to establish an in vitro model of cerebrovascular diseases[23-26].

SNW1, also known as SKIP, is a coactivator member of the SNW gene family[27]. SNW1, as a cofactor of transcription factors, can participate in precursor RNA splicing and recruit U2AF2 to mRNA[28]. Research has reported that SNW1 can interact with SNIP1 to regulate the stability of CyclinD1 mRNA[29]. Studies also showed that SNW1 has the mediated effects in the innate immune system. For example, SNW1 can participate in regulating the stress response of macrophages. Inhibition of SNW1 can also facilitate apoptosis of breast cancer cells[30] SNW1 is also involved in the regulation of the NF- $\mathrm{BB}$ signaling pathway[31]. It is reported that abnormal expression of SNW1 is relevant to rectal cancer, prostate cancer[32] and neuroblastoma[28]. Additionally, SNW1 overexpression can block normal neural crest development during embryonic development[33]. However, the influence and mechanism of SNW1 on CVD, especially BBB, have not been well elucidated. Therefore, our study aimed to further confirm the underlying influence and mechanism of SNW1 in CVD, principally BBB.

\section{Materials and Methods}

\section{Cell culture}


Mouse BMECs, from the Chinese Academy of Sciences (Shanghai, China), were hatched in RPMI1640 medium (Gibco, cat. no. 31800-014) with 10\% fetal bovine serum (FBS, Hyclone), $2 \mathrm{mmol} / \mathrm{l}$ glutamine (Sigma-Aldrich), $1 \mathrm{mmol} / \mathrm{l}$ sodium pyruvate (Sigma-Aldrich), $100 \mu \mathrm{g} / \mathrm{ml}$ streptomycin, and $50 \mu \mathrm{g} / \mathrm{ml}$ penicillin at $37^{\circ} \mathrm{C}$ and $5 \% \mathrm{CO}$. The cultured BMECs cells were given $0,50,100$, or $150 \mathrm{mg} / \mathrm{L}$ ox-LDL (Yiyuan Biotech Co., Ltd., Guangzhou, China) for $18 \mathrm{~h}$.

\section{Cell transfection}

siRNA against mouse SNW1 (si-SNW1) and the negative control (NC) were purchased from GenePharma (Shanghai GenePharma Co., Ltd. Shanghai, China). The sense sequence of si-SNW1 was 5'- GUCCAAAGACAAGGUCAUUTT-3' and the control siRNA was, 5'- UUCUCCGAACGUGUCACGUTT-3'. The empty vector (EV) and NLRP3-overexpressed plasmids were acquired from Hanbio Biotechnology (Shanghai, China). siRNA and plasmids were transfected to BMECs using Lipofectamine 3000 (Invitrogen, Carlsbad, CA) according to the manufacturer's protocol, and then incubated at $37^{\circ} \mathrm{C}$ for $48 \mathrm{~h}$. Finally, the cells were collected and prepared for the following experiments.

\section{Animals}

20 healthy Sprague-Dawley (SD) rats (9-week-old, weighing 240g-260g) were kept in the environment of the **experimental animal center at a temperature $\left(24^{\circ} \mathrm{C} \pm 2^{\circ} \mathrm{C}\right)$, humidity $(55 \% \pm 5 \%), 12 \mathrm{~h}$ of light and $12 \mathrm{~h}$ of darkness , and with sterile water. The SD rats were divided into the sham group $(n=5)$ and the high-fat $(H F)$ group $(n=15)$. The 
rats in sham and HF groups were fed with $25 \mathrm{~g}$ normal diet and $25 \mathrm{~g}$ HF diet every day for 12 weeks, respectively. Ethical permission for the animal experiments was obtained from the Ethics Committee of the $960^{\text {th }}$ hospital of PLA (approval no. 2020068). HF-fed rats were injected with EV (1.5 mL) or SNW1 knockdown lentivirus vector (1.5 $\mathrm{mL}$ ) in the tail. After 4 weeks of feeding, all rats fasted and serum, heart, brain tissue samples, and heart tissue were collected and stored at $-80{ }^{\circ} \mathrm{C}$ for examination.

\section{RT-qPCR assay}

Total RNAs were collected from the treated BMECs via Trizol method. After identification, Bestar qPCR RT kit (DBI Bioscience) was utilized for reverse transcription, and then SYBR Green PCR Master Mix (Applied Biosystems) was applied for gene amplification based the manufacturers instruction. The genes were quantified using the $2^{-\Delta \Delta \mathrm{CT}}$ method [34].

\section{Western blot}

We first used RIPA (1:100, cat no. P0013B) with a protease inhibitor (cocktail) to harvest total proteins. After centrifugation and re-suspension, the protein concentration was identified using BCAKit (Beyotime, China). $40 \mu \mathrm{g}$ proteins were separated through 10\% SDS-PAGE and transferred to PVDF membranes (Roche). After blocking, the proteins in membranes were placed in the corresponding primary antibody at $4{ }^{\circ} \mathrm{C}$ for the night, and then the secondary antibody (Abcam, cat. no. ab6802) for $1.5 \mathrm{~h}$. Subsequently, protein blots were viewed using the Electrochemiluminescence (ECL) system (Thermo Fisher Scientific). The primary antibodies were from Abcam. 


\section{Immunofluorescence (IF) assay}

After washing, the collected BMECs were fixed with $4 \%$ paraformaldehyde (Sigma Aldrich, cat. no. P6148) for $20 \mathrm{~min}$ and permeated with $0.5 \%$ Triton X-100 (Sigma Aldrich) for 20 min. After sealing with 5\% BSA (Sigma-Aldrich, cat no. A9418), cells were exposed to anti-SNW1 (1:50, Abcam) overnight at $4^{\circ} \mathrm{C}$, and the fluorescent second antibody (Abcam) for $1 \mathrm{~h}$. Then the nuclei were stained with $10 \mathrm{~g} / \mathrm{mL}$ DAPI, and the images were immediately observed using a fluorescent microscope.

\section{ELISA assay}

In accordance with the manufacturer's instructions, IL-18 and IL-33 levels in BMECs were quantified using the mouse IL-18 ELISA kit (Ek-Bioscience, cat. no. Ek-M20162) and mouse IL-33 ELISA kit (R\&D, cat. no. M3300), and IL-18 and IL-33 levels in rat serum were tested using the rat IL-18 ELISA kit (cat. no. YS03637B) and rat IL-33 ELISA kit (FineTest, cat. no. ER1903).

\section{Cholesterol efflux}

In line with the manufacturer's instructions, cholesterol efflux was determined using the cell-based cholesterol efflux assay kit (Abnova, cat. no. KA4542).

\section{CCK-8 assay}

BMECs $\left(5 \times 10^{3}\right.$ cells/well $)$ in 96 -well plate were treated following the specified processing method, and then processed with $15 \mu \mathrm{L} \mathrm{CCK}-8$ solution for $0,24,48$, and 72 h. After incubation for $3 \mathrm{~h}$, the absorbance was monitored with a microplate reader at $450 \mathrm{~nm}$. 


\section{Flow cytometry}

The processed BMECs were harvested through centrifugation and resuspended in the binding buffer. After counting, the BMECs had $5 \mu \mathrm{L}$ Annexin V-FITC and $5 \mu \mathrm{L}$ PI (BD Biosciences) added for $10 \mathrm{~min}$ in the dark. Then the apoptotic cells were tested by Flow cytometry (CytoFLEX, Beckman Coulter, Inc.) and analyzed with CELLQuest software (Becton Dickinson).

\section{Oil red O staining}

The aortic arch of the hearts of HF-fed rats ere collected and fixed with $4 \%$ paraformaldehyde (Sigma Aldrich, cat. no. P6148) for $30 \mathrm{~min}$, and then stained with 5\%

Oil Red O (Sigma) for $30 \mathrm{~min}$. After washing, the lipidtartalom in the images was observed by a microscope.

\section{Immunohistochemistry (IHC) assay}

After fixing, the samples were dehydrated, paraffin-embedded, and sliced into $4 \mu \mathrm{m}$ thick sections using a rotary microtome (Leica, GER). Then the sections were waxed in a baking machine at $42.5^{\circ} \mathrm{C}$ for $40 \mathrm{~min}$, processed with xylene, citrate buffer for $3 \mathrm{~min}$, and $\mathrm{H}_{2} \mathrm{O}_{2}$ for $10 \mathrm{~min}$. After washing, the sections were given primary antibodies at $4{ }^{\circ} \mathrm{C}$ for $12 \mathrm{~h}$, and second antibody at $37^{\circ} \mathrm{C}$ for $30 \mathrm{~min}$. The sections were had DAB solution added, Harris hematoxylin for 30s, and1\% hydrochloric acid alcohol. Then the sections were dehydrated, became transparent, and dried. The results were obtained using an inverted microscope (Nikon Eclipse TI-SR, Japan).

\section{Statistical Analysis}


Data expressed as mean \pm SD were analyzed using SPSS software 23.0 (SPSS Inc., Chicago, IL, USA). Data between the two groups were compared using the Student's t test. Data in multiple groups were compared using one-way ANOVA and Tamhane's. $P$ $<0.05$ denoted the results with statistical significance.

\section{Results}

\section{ox-LDL markedly upregulated SNW1 and NLRP3 in BMECs}

To investigate the possible mechanism of ox-LDL inducing oxidative injury of BMECs, BMECs were processed with $0,50,100$, or $150 \mathrm{mg} / \mathrm{L}$ ox-LDL. In accordance with RT-qPCR and Western blot results, it was found that SNW1 expression was prominently heightened in the ox-LDL group versus that in the blank group, and the increase of SNW1 expression was positively correlated with the dose of ox-LDL in BMECs $(P<0.05, P<0.01, P<0.001$, Fig. 1A-1C). Also, the results also found that as the concentration of ox-LDL increased, the protein level of NLRP3 was prominently elevated in BMECs $(P<0.05, P<0.01, P<0.001$, Fig. 1B and 1C). Therefore, our data showed that SNW1 and NLRP3 were connected with ox-LDL in BMECs.

\section{ox-LDL signally suppressed viability and increased cholesterol efflux in BMECs}

Next, the influences of ox-LDL on viability and cholesterol efflux in BMECs were determine. As CCK-8 results exhibited, cell viability was markly reduced in the ox-LDL treatment group relative to the blank group, in particular, in the $150 \mathrm{mg} / \mathrm{L}$ ox-LDL treatment group $(P<0.01, P<0.001$, Fig. 1D). In addition, the data showed 
that ox-LDL dramatically raised cholesterol efflux in BMECs, especially at $50 \mathrm{mg} / \mathrm{L}$ ox-LDL $(P<0.01, P<0.001$, Fig. 1E). Overall, these data found that ox-LDL induction can result in a significant inhibition of viability and a remarkable enhancement of cholesterol efflux in BMECs.

Silencing of SNW1 prominently raised cholesterol efflux and viability, and suppressed apoptosis and NLRP3 inflammasomes in ox-LDL-induced BMECs

Based on the upregulation of SNW1 in ox-LDL-induced BMECs, we speculated that SNW1 can affect the function of ox-LDL-induced BMECs. To confirm this hypothesis, we silenced SNW1 in ox-LDL-induced BMECs using siRNAs. The RT-qPCR and Western blot data showed that the transfection of SNW1 siRNAs could lead to a remarkable downregulation of SNW1 in BMECs, especially siRNA\#1 (Fig. 2A and 2B). Thus, SNW1 siRNA\#1 was applied in the subsequent experiments.

Subsequently, the IF results signified that silencing of SNW1 lowered SNW1 expression in BMECs, which had been increased by ox-LDL (Fig. 2C). Additionally, it was found that ox-LDL could markedly elevate IL-18 and IL-33 levels in BMECs, which could also be significantly attenuated by SNW1 knockdown in BMECs. In addition, cholesterol efflux was prominently enhanced in the SNW1 siRNAs group versus that in the NC group in ox-LDL-induced BMECs (Fig. 2D). Subsequently, flow cytometry was used to estimate the apoptotic change in ox-LDL-induced BMECs, and the data disclosed that relative to the blank group, cell apoptosis was notably 
enhanced in the ox-LDL treatment group, while this enhancement was markedly reversed by SNW1 knockdown in BMECs $(P<0.01$, Fig. $2 \mathbf{E})$. More importantly, it was found that ox-LDL treatment upregulated cholesterol efflux-related proteins (ABCA1 and ABCG1), which could also be further increased by SNW1 knockdown in BMECs. SNW1, NLRP3, and IL-1 $\beta$ expressions were markedly elevated in the ox-LDL group versus the blank group, while the expressions of these proteins could then be weakened by SNW1 knockdown in BMECs $(P<0.05, P<0.01$, Fig. 2F). Finally, the CCK- 8 data denoted that the viability of BMECs was markedly reduced in the ox-LDL group relative to the blank group, while the reduction of cell viability mediated by ox-LDL could be reversed by SNW1 silencing in BMECs $(P<0.05, P<0.001$, Fig. 2G). On the whole, these findings signified that SNW1 knockdown could dramatically protect BMECs injury induced by ox-LDL and repress NLRP3 inflammasome activation.

\section{Knockdown of SNW1 dramatically increased cholesterol efflux and viability, and prevented apoptosis and inflammasome activation by inhibiting NLRP3 in ox-LDL-induced BMECs}

Moreover, we studied whether NLRP3 can participate in the influence of SNW1 on ox-LDL-induced BMECs injury through a series of experiments. After SNW1 silencing, ox-LDL-treated BMECs were transfected with NLRP3 overexpression plasmids. First, Western blot data showed that upregulation of cholesterol efflux-related proteins (ABCA1 and ABCG1), which was mediated by SNW1 knockdown, could be 
significantly reversed by NLRP3 overexpression in ox-LDL-induced BMECs. NLRP3 overexpression also could dramatically elevate SNW1, NLRP3, IL-1 $\beta$ expressions, which had been downregulated by SNW1 silencing in ox-LDL-induced BMECs $(P<0.05, P<0.01$, Fig. 3A). Similarly, the IF results also demonstrated that the decrease of SNW1 expression, which was mediated by SNW1 knockdown, could be markedly attenuated by NLRP3 overexpression in ox-LDL-induced BMECs (Fig. 3B). In addition, it was found that knockdown of SNW1 increased cholesterol efflux, whereas this change was neutralized by SNW1 silencing in ox-LDL-induced BMECs $(P<0.05$, $P<0.01$, Fig. 3C). Simultaneously, it was found that overexpression of NLRP3 increased IL-18 and IL-33 levels in ox-LDL-induced BMECs, which had been decreased by knockdown of SNW1 $(P<0.05, P<0.01$, Fig. 3D and 3E). Furthermore, the data showed that inhibition of SNW1 silencing on apoptosis could be reversed by NLRP3 overexpression in ox-LDL-induced BMECs $(P<0.05$, Fig. 3F). SNW1 knockdown enhanced cell viability, and this enhancement was neutralized by NLRP3 overexpression in ox-LDL-induced BMECs $(P<0.05, P<0.001$, Fig. 3G). In summary, we found that the protective effect of SNW1 knockdown on ox-LDL-induced BMECs injury was achieved by inhibiting NLRP3.

\section{SNW1 knockdown elevated cholesterol efflux and reduced NLRP3 inflammasome activation in HF-fed rats}

Based on the above in vitro experiments, we further verified the role of SNW1 in HF-fed rats. We first injected HF-fed rats with SNW1 knockdown lentivirus and empty 
vector $(\mathrm{EV})$, respectively. As illustrated in Fig. 4A, the levels of SNW1 in heart and brain tissues, NLRP3 and IL-1 $\beta$ were elevated in the HF group in comparison with the sham group, while the elevated levels of these proteins could be significantly reduced by SNW1 knockdown in HF-fed rats. Similarly, HF feeding caused the expressions of ABCA1 and ABCG1 to rise in rats, while SNW1 knockdown further increased ABCA1 and ABCG1 expressions in HF-fed rats. Likewise, we found that IL-18 and IL-33 levels were higher in the HF group than that in the sham group, while HF-feeding mediated elevation of IL-18 and IL-33 levels could be partially reversed by SNW1 knockdown in rats (Fig. 4B and 4C). Next, Oil red $\mathrm{O}$ staining results showed that HF-feeding increased lipidtartalom in rats, while SNW1 knockdown markedly attenuated lipidtartalom, which was mediated by HF-feeding (Fig. 4D). Above all, the immunohistochemical analysis revealed that the protein levels of NLRP3 and SNW1 were dramatically increased in HF-fed rats versus that in sham rats, while this increase mediated by HF-feeding could be markedly lowed by SNW1 knockdown in the rat brain tissues (Fig. 4E and 4F). As a whole, we are convinced that SNW1 knockdown could also enhance cholesterol efflux and inhibit NLRP3 inflammasome activation in vivo.

\section{Discussion}

BMECs, as a class of highly specialized endothelial cells, are crucial in maintaining BBB function, dynamic balance of the cerebral microvascular system, and normal cerebral blood flow[11]. ox-LDL is an oxidative stress-damaging protein that can markedly cause intracellular lipid deposition[35]. In accordance with the reports, 
ox-LDL can activate NADPH oxidase (Nox) on BMECs and produce a mass of reactive oxygen species (ROS), leading to increased permeability of endothelial cells, which further causes lipid peroxidation to aggravates cellular injury[36, 37]. Also, ox-LDL can suppress nitric oxide synthase (NOS) activity in endothelial cells, accelerate nitric oxide (NO) degradation, and cause endothelial cell dysfunction[38]. ox-LDL can also induce expression of pro-inflammatory factors in endothelial cells and weaken the defensive effect of the antioxidant system. ox-LDL has been applied in multiple studies to induce BMEC injury, and Ox-LDL-induced BMEC cells were commonly used to construct cerebrovascular cell injury model in vitro[26, 39]. In our study, we also used ox-LDL to induce oxidative injury of BMECs, and the data signified that ox-LDL could prevent viability and accelerate cholesterol efflux in BMECs. Therefore, we studies the induction effect of OX-LDL on oxidative damage of BMECs. Interestingly, we also discovered that ox-LDL could prominently heighten expressions of SNW1 and NLRP3 in BMECs. Thus, we speculated that SNW1 and NLRP3 were involved in ox-LDL-induced oxidative injury in BMECs. SNW1, as a multifunctional protein involved in the regulation of multiple gene expression, has been reported to be associated with cell growth and cycle, apoptosis, as well as inflammatory response[28, 32, 40]. Currently, SNW1 has also been found to be relevant to diversified diseases, such as malignant pleural mesothelioma[41], hepatic carcinoma[42], breast cancer[43], and bladder cancer[44]. In our study, we first found that silencing SNW1 could prominently facilitate viability and prevent apoptosis of ox-LDL-induced BMECs. In 
addition, SNW1 knockdown could also enhance cholesterol efflux in ox-LDL-induced BMECs and HF-fed rats. Therefore, it was shown that knockdown of SNW1 has a significant mitigation effect on ox-LDL-induced oxidative stress injury in BMECs.

Recent study reveals that SNW1 were associated with inflammatory responses. SNW1 acts an important role in the pathogenesis of orheumatoid arthritis (RA) and might act as potential biomarker for RA[45]. SNW1 could inhibit influenza A virus (IAV) replication by inducing pro-inflammatory factors expression, such as IL-6, IFN$\beta$ [40]. More interestingly, a recent study has demonstrated that SNW1 is indispensable for the transcriptional elongation of NF- $\mathrm{BB}$ target genes such as the interleukin 8 (IL-8) and TNF genes[31].

Inflammasomes are multiprotein complexes, which were initiated and assembled by specific pattern recognition receptors (PRRs) of the cytoplasm[46, 47]. NLRP3 inflammasome, as the most widely studied inflammasome, is a protein complex composed of NLRP3, apoptosis-related speckled protein (ASC) and caspase-1[48]. While, there are evidences that NLRP3-inflammasomes are associated with BBB. For example, Ruscogenin can ameliorate BBB dysfunction induced by cerebral ischemia through NLRP3 Inflammasome[60]. Glibenclamide can attenuate the disrupted BBB via preventing NLRP3 inflammasome activation[61]. Hypertonic saline can reduce ischemic BBB permeability by mediating NLRP3 inflammasomes[62]. NLRP3 inflammasomes can induce the secretion and release of IL-1 $\beta$ and IL-18 by identifying pathogenic microorganisms, resulting in the inflammatory response[47, 49]. Also, 
NLRP3 inflammasomes can induce other inflammatory cytokines (such as IL-1 $\beta$ and IL18) and adhesion molecules, and further expand the inflammatory response in a cascade, causing tissue injury[50]. NLRP3 inflammasomes have been reported to play a significant role in brain injury[51]. For instance, isoliquiritigenin can ease brain injury via NLRP3 inflammasome[52] inhibition of IRE1 $\alpha$ which can affect hypoxic-ischemic brain injury by lowering NLRP3 inflammasome activation[53]. Interference of NLRP3-inflammasomes might be an effective method for neuroprotection after stroke[54].

Multiple studies showed that NF- $\mathrm{KB}$, as an upstream factor of NLRP3, can significantly upregulate NLRP3 [55-57]. Interestingly, SNW1 is a transcriptional regulator of the NF-kB pathway[31]. Here, we found that SNW1 knockdown can downregulate NLRP3 and IL-1 $\beta$ expressions in ox-LDL-induced BMECs and HF-fed rats, suggesting that SNW1 knockdown could prevent NLRP3 inflammasome activation, leading to decrease NLRP3-mediated IL-1 $\beta$ secretion. One likely possibility is that SNW1 regulated the NF- $\kappa \mathrm{B}$ pathway, and $\mathrm{NF}-\kappa \mathrm{B}$ up-regulated the NLRP3 inflammasome response. As a crucial nuclear transcription regulator, NF- $\kappa \mathrm{B}$ is involved in various physiological and pathological processes such as inflammation, stress response, cell proliferation, differentiation, apoptosis, and tissue damage and repair[58, 59]. In the current study, inhibition of SNW1 also could facilitate cholesterol efflux and proliferation, and suppress apoptosis of ox-LDL-induced BMECs by inhibiting NLRP3 inflammasome activation. 


\section{Conclusion}

Our study might be the first to uncover the function and mechanism of SNW1 knockdown in CVD. As the results showed, knockdown of SNW1 could dramatically attenuate ox-LDL-induced OGD/R-induced injury to BMECs by blocking NLRP3 inflammasome activation in vitro and in vivo. Therefore, this study expands our understanding of SNW1, suggesting that SNW1 knockdown might be a latent therapeutic target for CVD and the SNW1 inhibitor might be a potential therapeutic candidate for the treatment of CVD.

\section{Funding Statement}

The author(s) received no specific funding for this study.

\section{Conflicts of Interest}

The authors declare that they have no conflicts of interest to report regarding the present study.

\section{Acknowledgement}

Thanks to Dr. Edward C. Mignot, Shandong University, for linguistic advice.

\section{References}

1. Portegies M L, Koudstaal P J, Ikram M A. Cerebrovascular disease. Handb Clin Neurol 2016; 138 : 239-61.

2. Caprio F Z, Sorond F A. Cerebrovascular Disease: Primary and Secondary Stroke Prevention. Med Clin North Am 2019; 103: 295-308.

3. Letra L, Sena C. Cerebrovascular Disease: Consequences of Obesity-Induced Endothelial Dysfunction. Adv Neurobiol 2017; 19: 163-189.

4. Li B, Chen S, Qi X, et al. The numerical study on specialized treatment strategies of enhanced external counterpulsation for cardiovascular and cerebrovascular disease. Med Biol Eng Comput 2018; 56: 1959-1971.

5. Yongjun W. The particular issues of prevention andcontrol of cerebrovascular disease in China. Chinese ence Bulletin 2016; 61: 2020-2026. 
6. Bösel J, Amiri H. The utility of cardiovascular drugs in the treatment of cerebrovascular disease. Curr Opin Investig Drugs 2010; 11: 1015-24.

7. Nikolic D, Giglio R V, Rizvi A A, et al. Liraglutide Reduces Carotid Intima-Media Thickness by Reducing Small Dense Low-Density Lipoproteins in a Real-World Setting of Patients with Type 2 Diabetes: A Novel Anti-Atherogenic Effect. Diabetes Ther 2021; 12: 261-274.

8. Rizzo $\mathrm{M}$, Abate $\mathrm{N}$, Chandalia $\mathrm{M}$, et al. Liraglutide reduces oxidative stress and restores heme oxygenase- 1 and ghrelin levels in patients with type 2 diabetes: a prospective pilot study. J Clin Endocrinol Metab 2015; 100: 603-6.

9. Rizzo M, Nikolic D, Patti A M, et al. GLP-1 receptor agonists and reduction of cardiometabolic risk: Potential underlying mechanisms. Biochim Biophys Acta Mol Basis Dis 2018; 1864: 2814-2821.

10. Persidsky Y, Ramirez S H, Haorah J, Kanmogne G D. Blood-brain barrier: structural components and function under physiologic and pathologic conditions. J Neuroimmune Pharmacol 2006; 1: 223-36.

11. Obermeier B, Verma A, Ransohoff R M. The blood-brain barrier. Handb Clin Neurol 2016; 133: 39-59.

12. Varatharaj A, Galea I. The blood-brain barrier in systemic inflammation. Brain Behav Immun 2017; 60: 1-12.

13. Jumnongprakhon P, Govitrapong P, Tocharus C, Tocharus J. Melatonin promotes blood-brain barrier integrity in methamphetamine-induced inflammation in primary rat brain microvascular endothelial cells. Brain Res 2016; 1646: 182-192.

14. Bernstein D L, Zuluaga-Ramirez V, Gajghate S, et al. miR-98 reduces endothelial dysfunction by protecting blood-brain barrier (BBB) and improves neurological outcomes in mouse ischemia/reperfusion stroke model. J Cereb Blood Flow Metab 2020; 40: 1953-1965.

15. Rom S, Zuluaga-Ramirez V, Reichenbach N L, et al. Secoisolariciresinol diglucoside is a blood-brain barrier protective and anti-inflammatory agent: implications for neuroinflammation. J Neuroinflammation 2018; 15: 25.

16. Deng S, Liu H, Qiu K, You H, Lei Q, Lu W. Role of the Golgi Apparatus in the Blood-Brain Barrier: Golgi Protection May Be a Targeted Therapy for Neurological Diseases. Mol Neurobiol 2018; 55: 4788-4801.

17. Sweeney M D, Sagare A P, Zlokovic B V. Blood-brain barrier breakdown in Alzheimer disease and other neurodegenerative disorders. Nat Rev Neurol 2018; 14: 133-150.

18. Griffin A D, Turtzo L C, Parikh G Y, et al. Traumatic microbleeds suggest vascular injury and predict disability in traumatic brain injury. Brain 2019; 142: 3550-3564.

19. Uno M, Kitazato K T, Nishi K, Itabe H, Nagahiro S. Raised plasma oxidised LDL in acute cerebral infarction. J Neurol Neurosurg Psychiatry 2003; 74: 312-6.

20. Rouhl R P, Van Oostenbrugge R J, Theunissen R O, et al. Autoantibodies against oxidized low-density lipoprotein in cerebral small vessel disease. Stroke 2010; 41: 2687-9.

21. Uno M, Harada M, Takimoto $O$, et al. Elevation of plasma oxidized LDL in acute stroke patients is associated with ischemic lesions depicted by DWI and predictive of infarct enlargement. Neurol Res 2005; 27: 94-102.

22. Farrall A J, Wardlaw J M. Blood-brain barrier: ageing and microvascular disease--systematic review and meta-analysis. Neurobiol Aging 2009; 30: 337-52. 
23. Shie F S, Neely M D, Maezawa I, et al. Oxidized low-density lipoprotein is present in astrocytes surrounding cerebral infarcts and stimulates astrocyte interleukin-6 secretion. Am J Pathol 2004; 164: 1173-81.

24. Zhang $Q$, Liu C, Li Q, Li J, Wu Y, Liu J. MicroRNA-25-5p counteracts oxidized LDL-induced pathological changes by targeting neuronal growth regulator 1 (NEGR1) in human brain micro-vessel endothelial cells. Biochimie 2019; 165: 141-149.

25. Lin Y L, Chang H C, Chen T L, et al. Resveratrol protects against oxidized LDL-induced breakage of the blood-brain barrier by lessening disruption of tight junctions and apoptotic insults to mouse cerebrovascular endothelial cells. J Nutr 2010; 140: 2187-92.

26. Wang X, Mao R, Chen W. FSD-C10 Shows Therapeutic Effects in Suppressing oxidized low-density lipoprotein (ox-LDL)-Induced Human Brain Microvascular Endothelial Cells Apoptosis via Rho-Associated Coiled-Coil Kinase (ROCK)/Mitogen-Activated Protein Kinase (MAPK) Signaling. Med Sci Monit 2018; 24: 5509-5516.

27. Olgun C E, Muyan M. SNW1 (SNW domain containing 1). Atlas of Genetics and Cytogenetics in Oncology and Haematology 2018; 22.

28. Hong M, He J, Li S. SNW1 regulates Notch signaling in neuroblastoma through interacting with RBPJ. Biochem Biophys Res Commun 2019; 509: 869-876.

29. Bracken C P, Wall S J, Barré B, Panov K I, Ajuh P M, Perkins N D. Regulation of cyclin D1 RNA stability by SNIP1. Cancer Res 2008; 68: 7621-8.

30. Sato N, Maeda M, Sugiyama M, et al. Inhibition of SNW1 association with spliceosomal proteins promotes apoptosis in breast cancer cells. Cancer Med 2015; 4: 268-77.

31. Verma S, De Jesus P, Chanda S K, Verma I M. SNW1, a Novel Transcriptional Regulator of the NF-kB Pathway. Mol Cell Biol 2019; 39.

32. Hoflmayer D, Willich C, Hube-Magg C, et al. SNW1 is a prognostic biomarker in prostate cancer. Diagn Pathol 2019; 14: 33.

33. Wu M Y, Ramel M C, Howell M, Hill C S. SNW1 is a critical regulator of spatial BMP activity, neural plate border formation, and neural crest specification in vertebrate embryos. PLoS Biol 2011; 9: e1000593.

34. Livak K J, Schmittgen T D. Analysis of relative gene expression data using real-time quantitative PCR and the 2(-Delta Delta C(T)) Method. Methods 2001; 25: 402-8.

35. Kattoor A J, Kanuri S H, Mehta J L. Role of Ox-LDL and LOX-1 in Atherogenesis. Curr Med Chem 2019; 26: 1693-1700.

36. Najafi M, Roustazadeh A, Alipoor B. Ox-LDL Particles: Modified Components, Cellular Uptake, Biological Roles and Clinical Assessments. Cardiovasc Hematol Disord Drug Targets 2011; 11: 119-28.

37. Pirillo A, Norata G D, Catapano A L. LOX-1, OxLDL, and atherosclerosis. Mediators Inflamm 2013; 2013: 152786.

38. Zhao J, Zhang Q, Liu J, et al. Effect of Endomorphins on HUVECs Treated by ox-LDL and Its Related Mechanisms. J Diabetes Res 2016; 2016: 9741483.

39. Rochfort K D, Cummins P M. Cytokine-mediated dysregulation of zonula occludens-1 properties in human brain microvascular endothelium. Microvasc Res 2015; 100: 48-53.

40. Zhang $Q$, Liang T, Gu S, Ye Y, Liu S. SNW1 interacts with IKK $\gamma$ to positively regulate antiviral innate immune responses against influenza A virus infection. Microbes Infect 2020; 22: 576-584. 
41. Türkcü G, Alabalik U, Keles A N, et al. Comparison of SKIP expression in malignant pleural mesotheliomas with Ki-67 proliferation index and prognostic parameters. Pol J Pathol 2016; 67: 108-13.

42. Liu G, Huang X, Cui X, et al. High SKIP expression is correlated with poor prognosis and cell proliferation of hepatocellular carcinoma. Med Oncol 2013; 30: 537.

43. Liu X, Ni Q, Xu J, et al. Expression and prognostic role of SKIP in human breast carcinoma. J Mol Histol 2014; 45: 169-80.

44. Wang $L$, Zhang M, Wu Y, et al. SKIP expression is correlated with clinical prognosis in patients with bladder cancer. Int J Clin Exp Pathol 2014; 7: 1695-701.

45. Sabir J S M, El Omri A, Banaganapalli B, et al. Dissecting the Role of NF-kb Protein Family and Its Regulators in Rheumatoid Arthritis Using Weighted Gene Co-Expression Network. Front Genet 2019; 10: 1163.

46. Broz P, Dixit V M. Inflammasomes: mechanism of assembly, regulation and signalling. Nat Rev Immunol 2016; 16: 407-20.

47. Guo N, Zhang J. Interleukin-17 promotes ovarian carcinoma SKOV3 cells via MTA1-induced epithelial-to-mesenchymal transition. European Journal of Gynaecological Oncology 2020; 41: 70-74.

48. Kelley N, Jeltema D, Duan Y, He Y. The NLRP3 Inflammasome: An Overview of Mechanisms of Activation and Regulation. Int J Mol Sci 2019; 20.

49. Sutterwala F S, Haasken S, Cassel S L. Mechanism of NLRP3 inflammasome activation. Ann N Y Acad Sci 2014; 1319: 82-95.

50. Zhong Z, Sanchez-Lopez E, Karin M. Autophagy, NLRP3 inflammasome and auto-inflammatory/immune diseases. Clin Exp Rheumatol 2016; 34: 12-6.

51. Gong Z, Pan J, Shen Q, Li M, Peng Y. Mitochondrial dysfunction induces NLRP3 inflammasome activation during cerebral ischemia/reperfusion injury. J Neuroinflammation 2018; 15: 242.

52. Zeng J, Chen $\mathrm{Y}$, Ding $\mathrm{R}$, et al. Isoliquiritigenin alleviates early brain injury after experimental intracerebral hemorrhage via suppressing ROS- and/or NF-KB-mediated NLRP3 inflammasome activation by promoting Nrf2 antioxidant pathway. J Neuroinflammation 2017; 14: 119.

53. Chen D, Dixon B J, Doycheva D M, et al. IRE1 $\alpha$ inhibition decreased TXNIP/NLRP3 inflammasome activation through miR-17-5p after neonatal hypoxic-ischemic brain injury in rats. J Neuroinflammation 2018; 15: 32.

54. Ismael S, Zhao L, Nasoohi S, Ishrat T. Inhibition of the NLRP3-inflammasome as a potential approach for neuroprotection after stroke. Sci Rep 2018; 8: 5971.

55. An $\mathrm{Y}$, Zhang $\mathrm{H}$, Wang $\mathrm{C}$, et al. Activation of ROS/MAPKs/NF-KB/NLRP3 and inhibition of efferocytosis in osteoclast-mediated diabetic osteoporosis. Faseb j 2019; 33: 12515-12527.

56. Yi $\mathrm{H}$, Peng $\mathrm{R}$, Zhang $\mathrm{L} Y$, et al. LincRNA-Gm4419 knockdown ameliorates NF-kB/NLRP3 inflammasome-mediated inflammation in diabetic nephropathy. Cell Death Dis 2017; 8: e2583.

57. Yu X, Lan P, Hou X, et al. HBV inhibits LPS-induced NLRP3 inflammasome activation and IL-1 $\beta$ production via suppressing the NF-KB pathway and ROS production. J Hepatol 2017; 66: 693-702.

58. Mitchell J P, Carmody R J. NF-KB and the Transcriptional Control of Inflammation. Int Rev Cell Mol Biol 2018; 335: 41-84.

59. Lin T H, Pajarinen J, Lu L, et al. NF-kB as a Therapeutic Target in Inflammatory-Associated Bone 
Diseases. Adv Protein Chem Struct Biol 2017; 107: 117-154.

60. Cao G, Jiang N, Hu Y, et al. Ruscogenin Attenuates Cerebral Ischemia-Induced Blood-Brain Barrier Dysfunction by Suppressing TXNIP/NLRP3 Inflammasome Activation and the MAPK Pathway. Int J Mol Sci 2016; 17.

61. Xu F, Shen G, Su Z, He Z, Yuan L. Glibenclamide ameliorates the disrupted blood-brain barrier in experimental intracerebral hemorrhage by inhibiting the activation of NLRP3 inflammasome. Brain Behav 2019; 9: e01254.

62. Wang Q S, Ding H G, Chen S L, et al. Hypertonic saline mediates the NLRP3/IL-1 $\beta$ signaling axis in microglia to alleviate ischemic blood-brain barrier permeability by downregulating astrocyte-derived VEGF in rats. CNS Neurosci Ther 2020; 26: 1045-57.

\section{Figure legends}




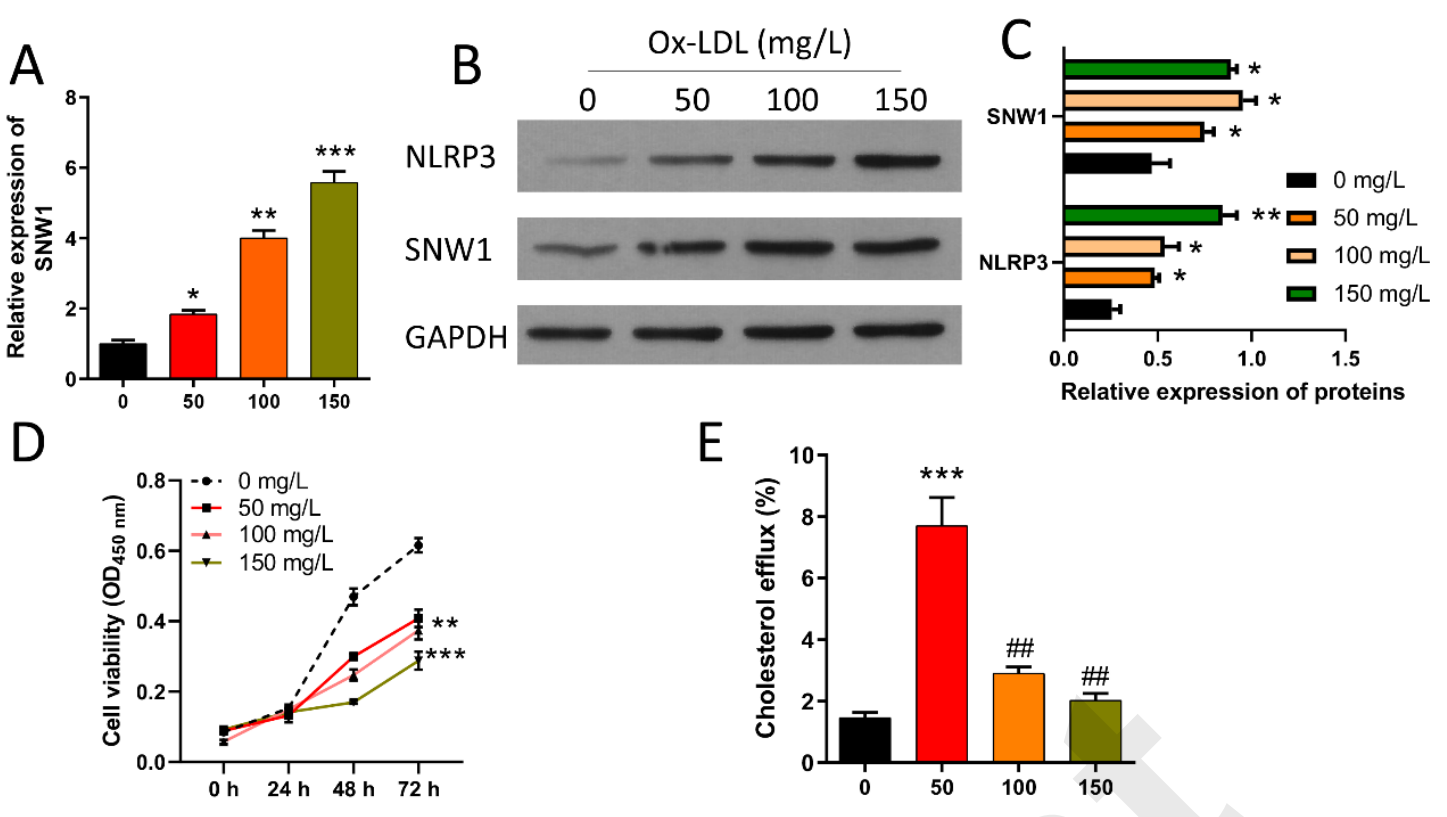

Fig. 1. ox-LDL markedly upregulated SNW1 and NLRP3, suppressed viability, and increased cholesterol efflux in BMECs. (A) SNW1 expression was monitored via RT-qPCR in BMECs after processing with 0, 50, 100, or $150 \mathrm{mg} / \mathrm{L}$ ox-LDL. (B) Western blot identification of SNW1 and NLRP3 proteins in ox-LDL-treated BMECs. (C) Quantitative analysis of SNW1 and NLRP3 expressions on account of the protein gray values. (D) ox-LDL-mediated cell viability was certified through CCK-8 in BMECs. (E) After 0, 50, 100, or $150 \mathrm{mg} / \mathrm{L}$ ox-LDL, cholesterol efflux was validated using the corresponding kit. $* P<0.05, * * P<0.01, * * * P<0.001$. 


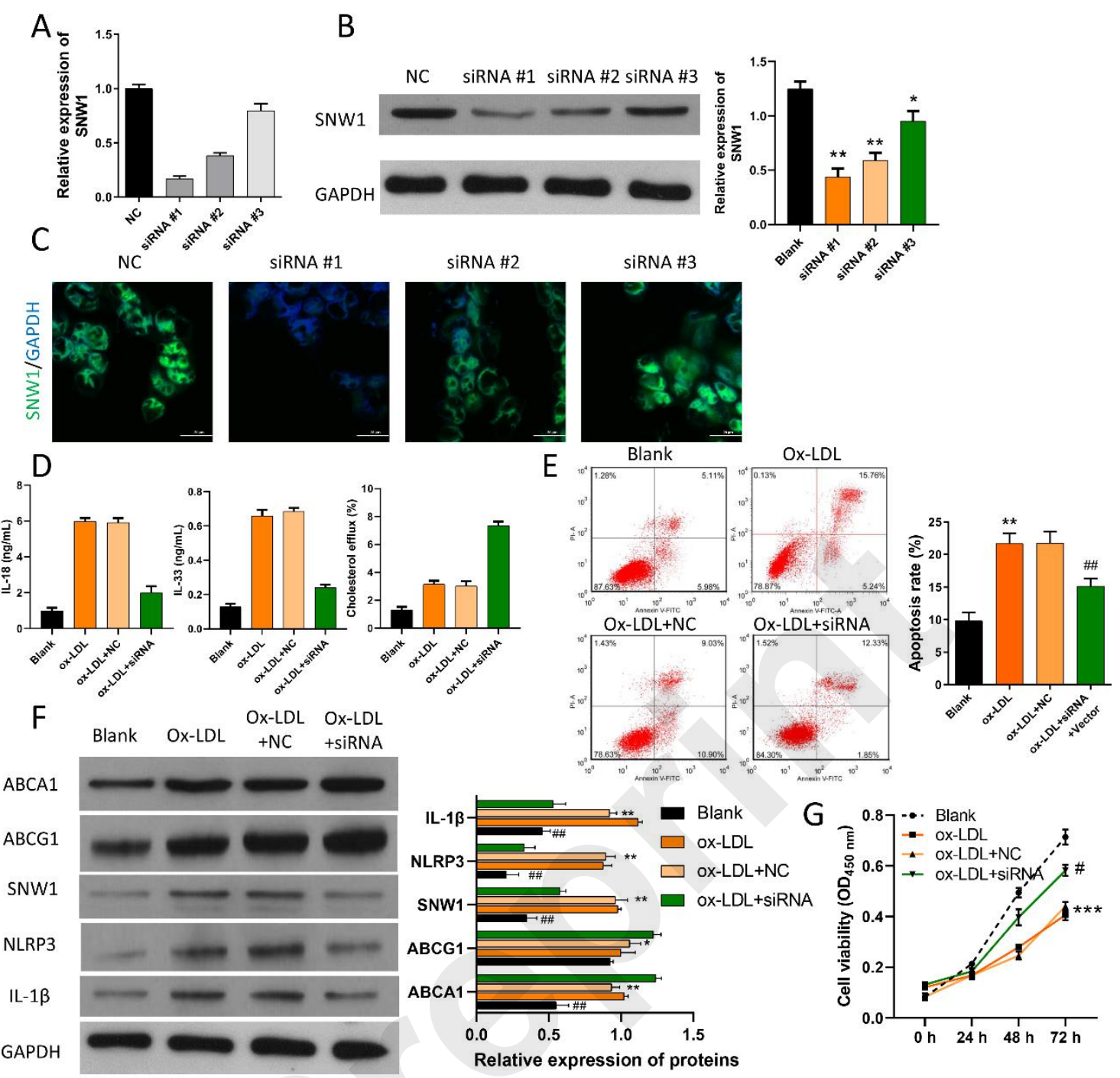

Fig. 2. Silencing of SNW1 prominently raised cholesterol efflux and viability, and suppressed apoptosis and NLRP3 inflammasomes in ox-LDL-induced BMECs. The silencing effect of SNW1 was identified via RT-qPCR (A) and Western blot (B) in BMECs after transfection with siRNA\#1, \#2, and \#3. (C) The expression of SNW1 was verified via IF assay in ox-LDL-induced BMECs, which were transfected with si-NC and si-SNW1 (siRNA\#1). Magnification, $\times 200$; Scale bar=20 $\mu$ m. (D) The impacts of SNW1 knockdown on IL-18, IL-33, and cholesterol efflux were assessed by applying 
respective kits in ox-LDL-treated BMECs. (E) Change in the apoptosis of BMECs was determined via flow cytometry after SNW1 knockdown. (F) Expression changes of ABCA1, ABCG1, SNW1, NLRP3, and IL-1 $\beta$ proteins were verified with Western blot in ox-LDL-mediated BMECs after SNW1 silencing. (G) After transfection with SNW1 siRNAs, cell viability was tested using CCK-8 in ox-LDL-treated BMECs. $* P<0.05$, $* * P<0.01, * * * P<0.001$ vs. the blank group; $\# P<0.05, \# \# P<0.01$ vs. the ox-LDL+NC group. 


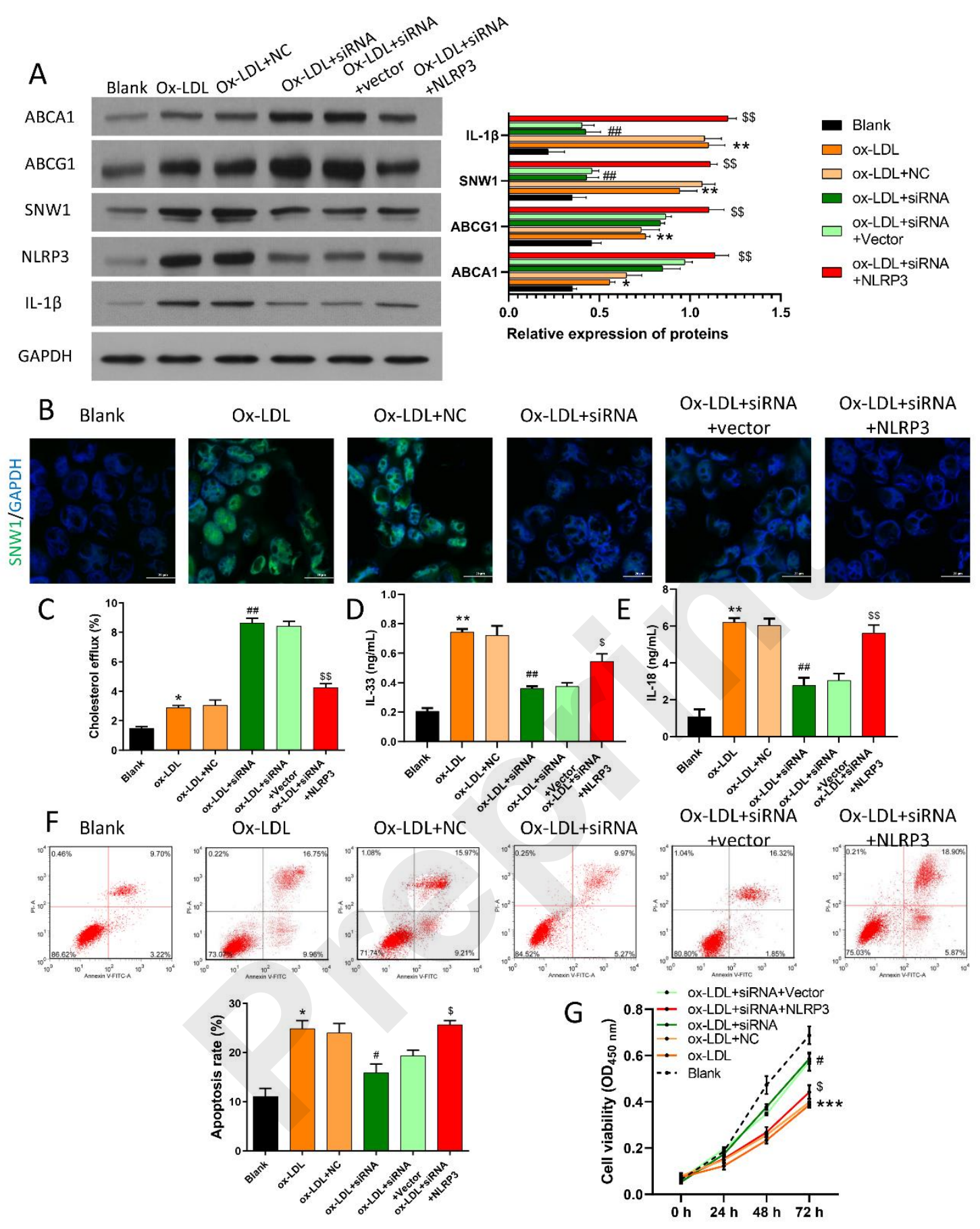

Fig. 3. Knockdown of SNW1 dramatically increased cholesterol efflux and viability, and prevented apoptosis and inflammasome activation by inhibiting NLRP3 in ox-LDL-induced BMECs. ox-LDL-treated BMECs were transfected with SNW1 siRNAs or/and NLRP3-overexpressed plasmid, respectively. (A) Detection of $\mathrm{F}$ 
ABCA1, ABCG1, SNW1, NLRP3, and IL-1 $\beta$ proteins through Western blot in BMECs in each group. The protein levels of each group with Western blot results were quantitatively analyzed. (B) Identification of SNW1 expression and location via IF assay in the processed BMECs. (C) After SNW1 knockdown or/and NLRP3 overexpression, cholesterol efflux was analyzed using the kit in BMECs. (D) IL-18 and (E) IL-33 levels were tested with ELISA kits. (F) Flow cytometry was used for apoptosis detection in the disposed BMECs. (G) After transfection, CCK-8 was used to monitor the viability of ox-LDL-treated BMECs. $* P<0.05$, $* * P<0.01, * * * P<0.001$ vs. the blank group; $\# P<0.05$, \#\#P<0.01 vs. the ox-LDL+NC group; $\$ P<0.05, \$ \$ P<0.01$ vs. the ox-LDL+siRNA+Vector group. 


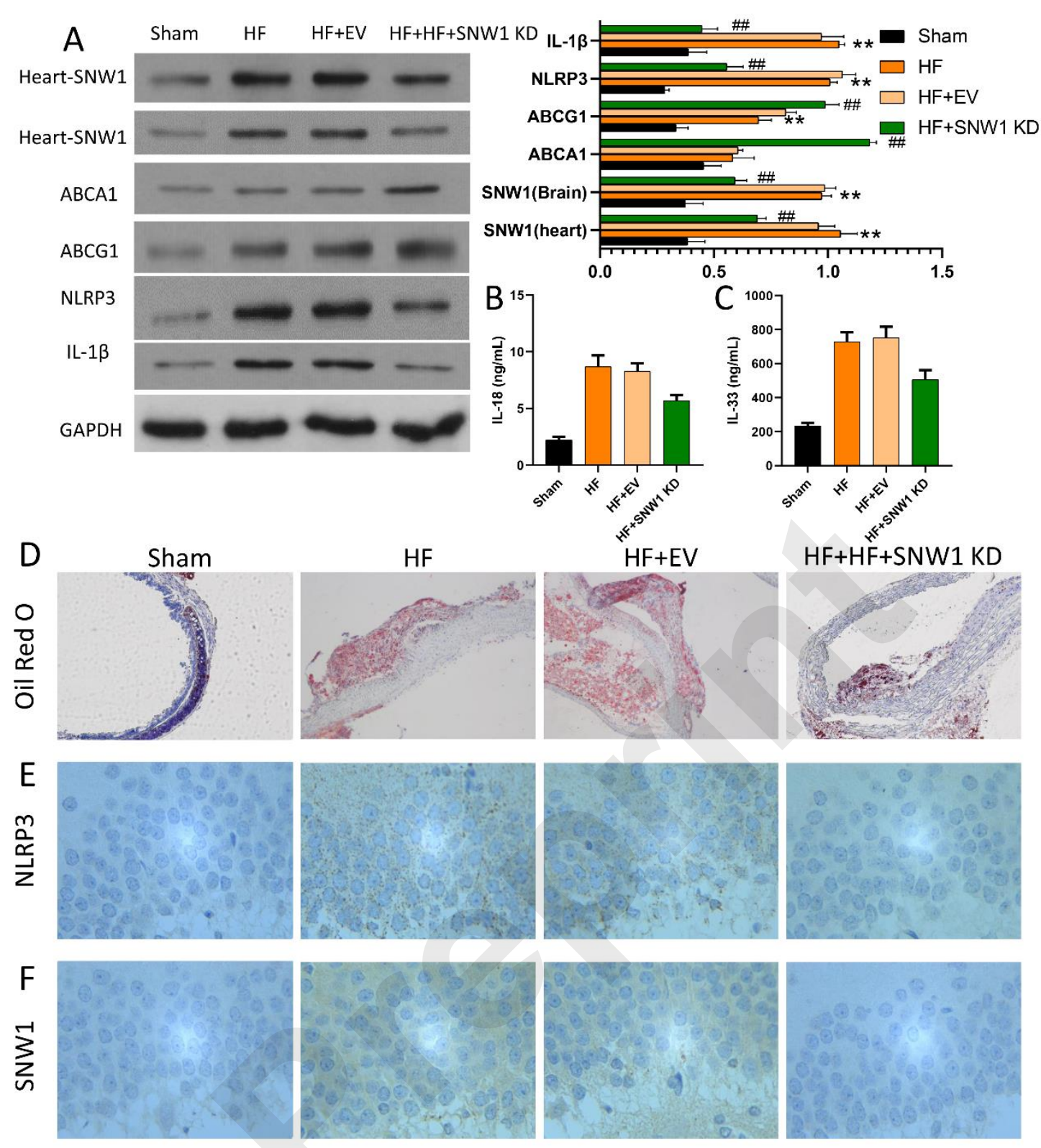

Fig. 4. SNW1 knockdown elevated cholesterol efflux and reduced NLRP3 inflammasome activation in HF-fed rats. Rats fed with high-fat were injected with empty vector (EV) or SNW1 knockdown lentivirus, respectively. (A) SNW1, ABCA1, ABCG1, NLRP3, and IL-1 $\beta$ levels were determined through Western blot in HF-fed rats after SNW1 knockdown. Each protein was quantitatively calculated using each gray value. The concentrations of IL-18 (B) and IL-33 (C) were identified via ELISA kits. (D) 
After SNW1 knockdown, the lipidtartalom was evaluated by Oil red O staining in the aortic arch of the hearts of HF-fed rats. The expressions NLRP3 (E) and SNW1 (F) were confirmed using immunohistochemical analysis in brain tissues of HF-fed rats after SNW1 knockdown. 


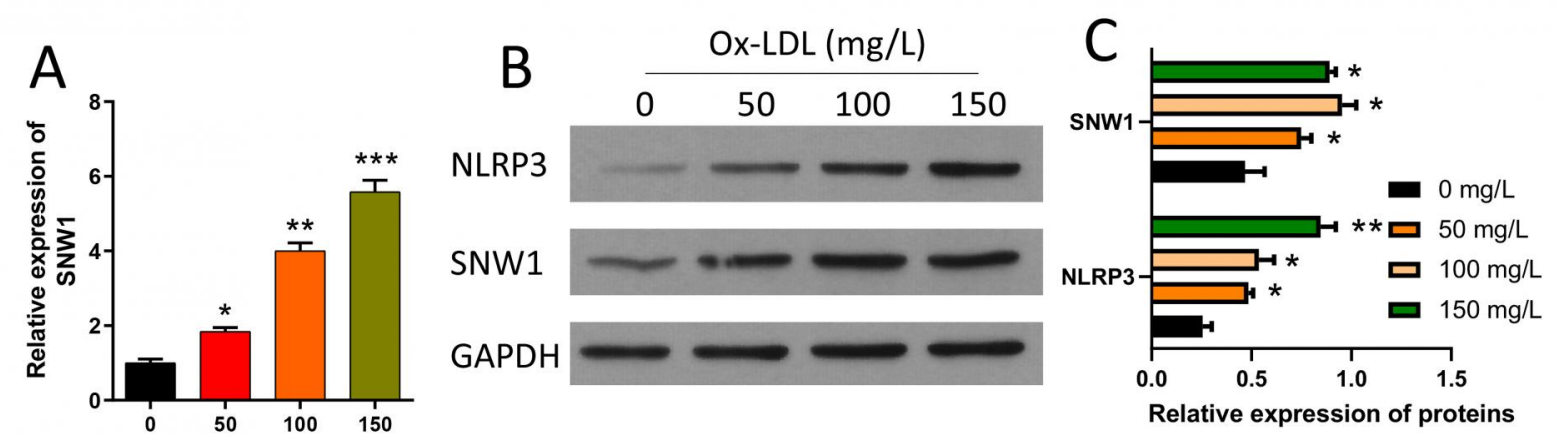

D

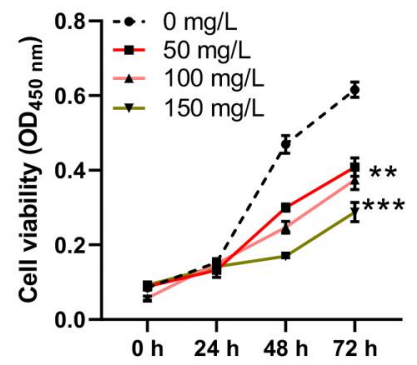

$E$

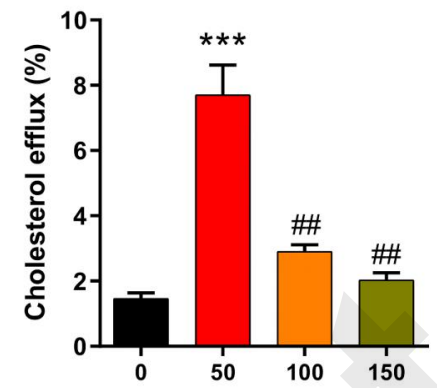



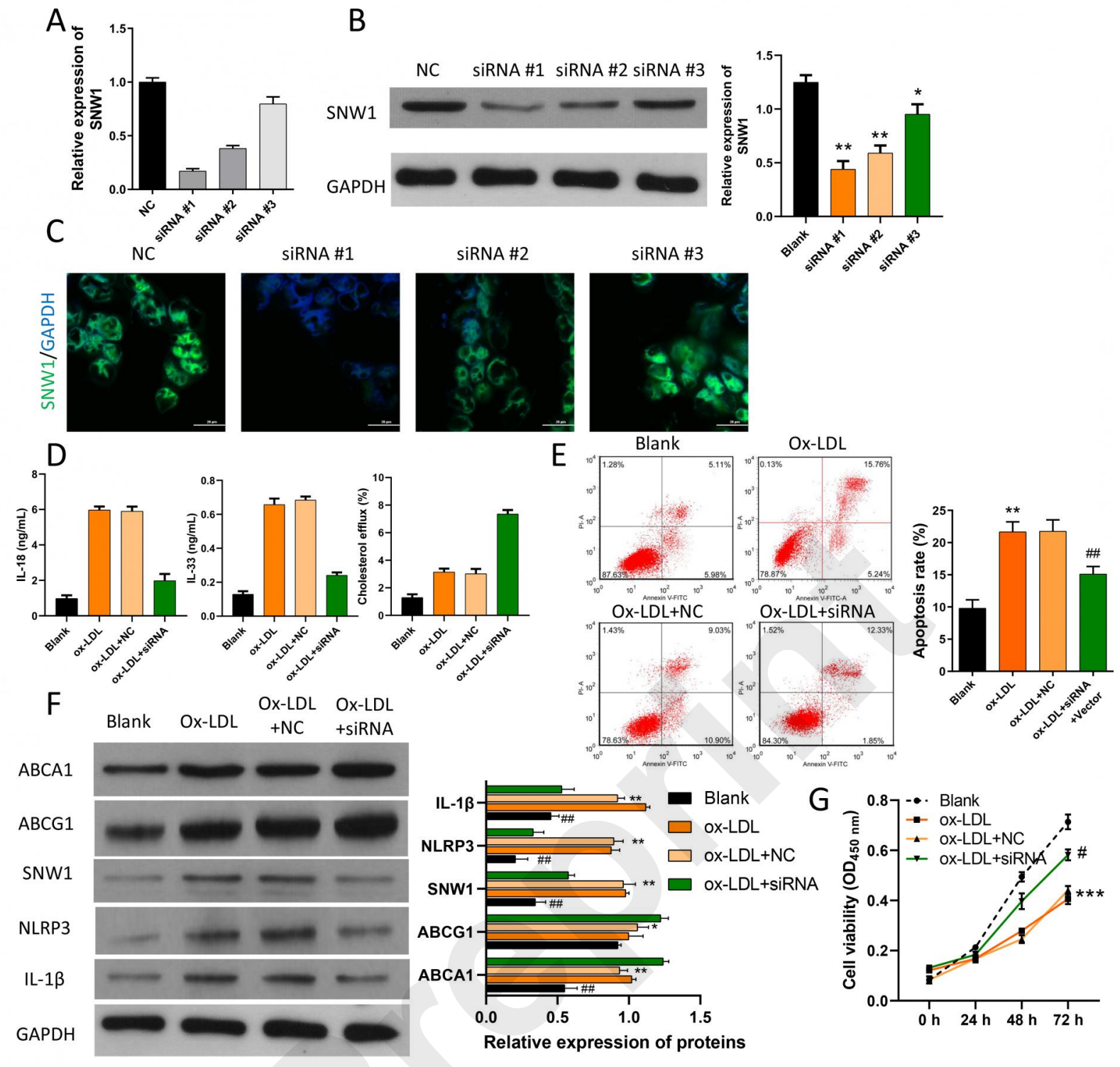


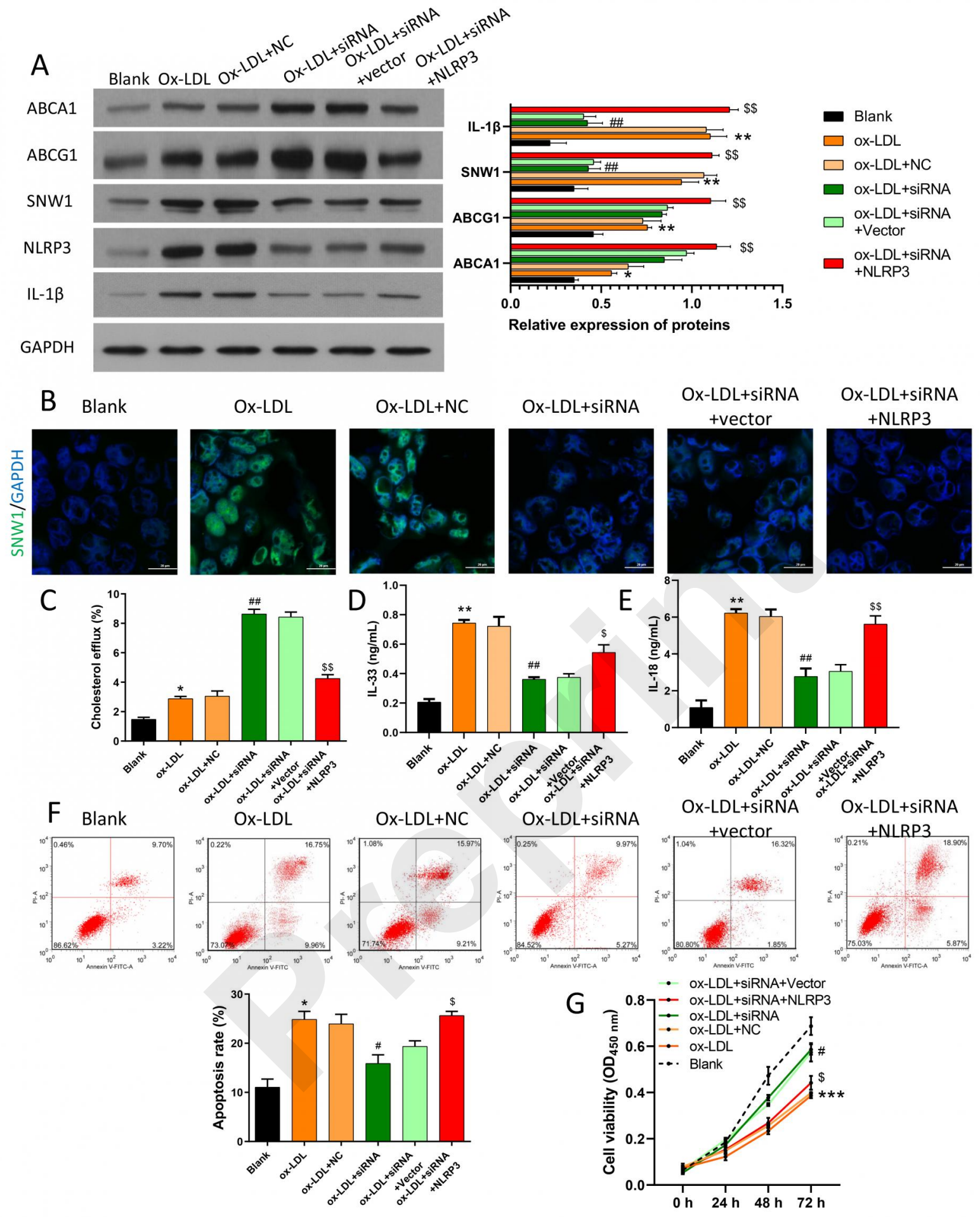




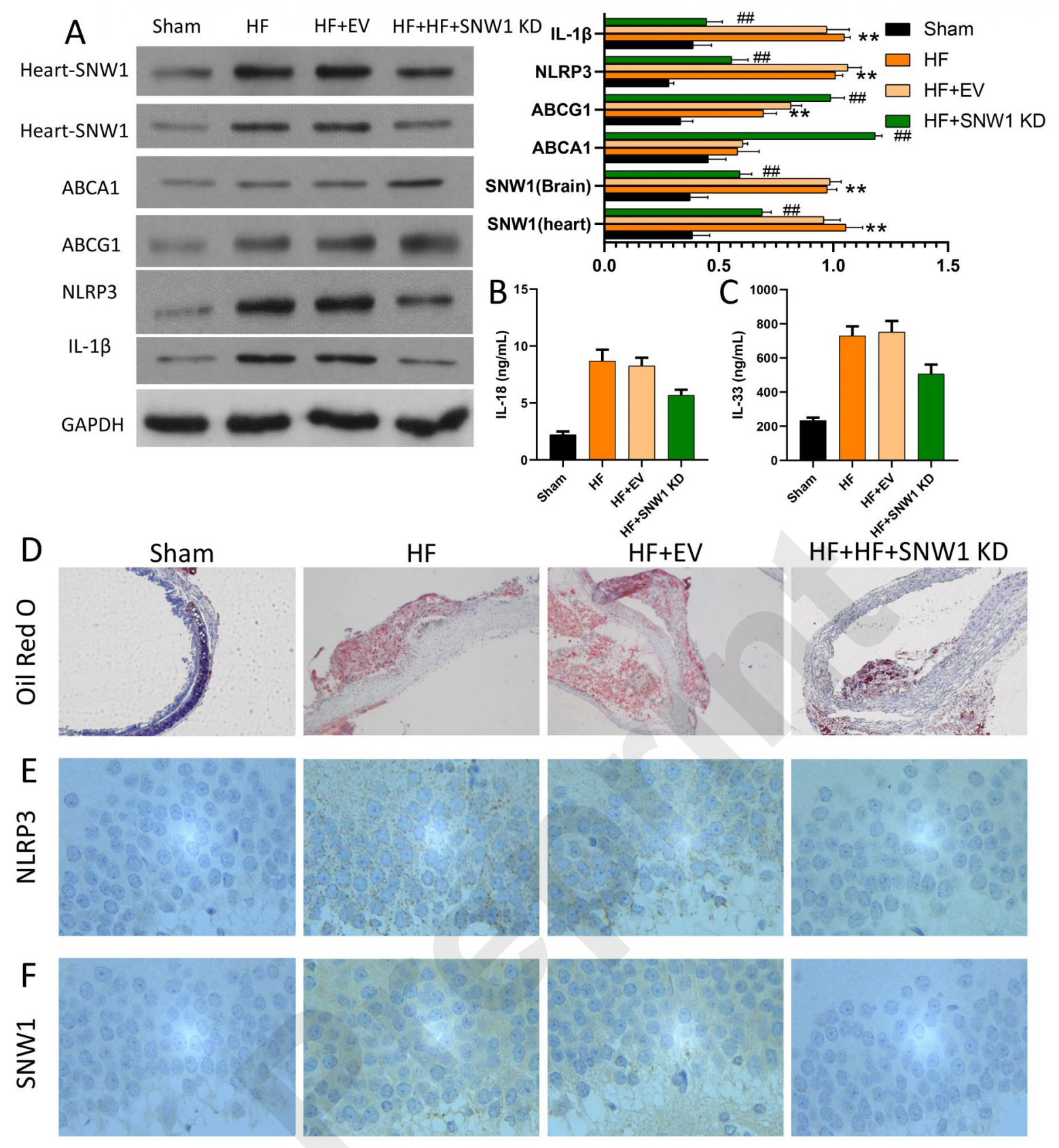

